International Mathematical Forum, 2, 2007, no. 14, 689 - 698

\title{
Third-order iterative methods free from second derivative
}

\author{
Kou Jisheng ${ }^{1}$ and Li Yitian \\ State Key Laboratory of Water Resources and \\ Hydropower Engineering Sciences \\ Wuhan University, Wuhan 430072, China \\ Liu Dingyou and He Julin \\ School of Mathematics and Statistics \\ Wuhan University, Wuhan 430072, China
}

\begin{abstract}
In this paper, we present a class of new iterative methods, in which $f^{\prime}(x)=0$ in some points is permitted. Analysis of convergence shows that the new methods are cubically convergent. Per iteration the new methods require one evaluation of the function and two of its first derivative, but no evaluations of its second derivative. Thus, the new methods have definite practical utility, which is demonstrated by numerical examples.
\end{abstract}

Mathematics Subject Classification: 41A25, 65D99

Keywords: Newton method, Third-order convergence, Non-linear equations, Iterative method

\footnotetext{
${ }^{1}$ Corresponding author. e-mail: koujisheng@yahoo.com.cn (J. Kou ).
} 


\section{Introduction}

Solving non-linear equations is one of the most important problems in numerical analysis. In this paper, we consider iterative methods to find a simple root of a non-linear equation $f(x)=0$, where $f: D \subset \mathbb{R} \rightarrow \mathbb{R}$ for an open interval $D$ is a scalar function. Newton method is an important and basic method [1], which converges quadratically. Recently, a class of new modified Newton methods with cubic convergence has been developed [2-8]. These methods are very important and interesting because they do not require the second derivative of $f(x)$, different from the methods that involve second derivatives (for a recent review of the latter methods, see [9]).

However, we know that Newton method require initial approximations sufficiently close to the required root. Especially the condition $f^{\prime}(x) \neq 0$ in a neighborhood of the required root is severe indeed for convergence of Newton method and its applications is restricted. Similar to Newton method, these modified Newton methods have also such problem which restricts their applications.

For resolving the above problem of Newton method, Wu proposes a class of Newton-like methods [10]

$$
x_{n+1}=x_{n}-\frac{f\left(x_{n}\right)}{f^{\prime}\left(x_{n}\right)+\lambda_{n} f\left(x_{n}\right)},
$$

where $\lambda_{n} \in \mathbb{R}, 0<\left|\lambda_{n}\right|<+\infty$ and $\lambda_{n}$ is chosen such that the corresponding function values $\lambda_{n} f\left(x_{n}\right)$ and $f^{\prime}\left(x_{n}\right)$ have the same signs. This class of methods converges quadratically under the condition $\lambda_{n} f\left(x_{n}\right)+f^{\prime}\left(x_{n}\right) \neq 0$, while $f^{\prime}\left(x_{n}\right)=0$ in some points is permitted. However, it is to be expected resolving such problem of the above modified Newton methods. As these modified Newton methods are preferable to Newton method in many cases [3, 5], for expanding their applications, it is very necessary to remove the severe condition $f^{\prime}(x) \neq 0$ in a neighborhood of the required root.

The main aim of this paper is to extend the results in [10] to the above modified Newton methods with cubic convergence. We firstly present a new third-order iterative method with second derivative, which permits $f^{\prime}(x)=0$ in some points. Then, by replacing the second derivative with a finite differ- 
ence quotient between the first derivatives, we obtain a class of new iterative methods with cubic convergence, in which the severe condition $f^{\prime}(x) \neq 0$ is removed. As particular cases, we introduce two methods of this class of methods, which may be viewed as variants of the above modified Newton methods. Numerical experiments show that these new methods are efficient alternatives in many cases where some modified Newton methods are not successful.

\section{Third-order method with second derivative}

To derive the proposed methods, we firstly consider a new iterative method with second derivative

$$
x_{n+1}=x_{n}-\frac{\left(L_{f}\left(x_{n}\right)+f^{\prime}\left(x_{n}\right)+\mu_{n} f\left(x_{n}\right)\right) f\left(x_{n}\right)}{\left(L_{f}\left(x_{n}\right)+f^{\prime}\left(x_{n}\right)+\frac{1}{2} \mu_{n} f\left(x_{n}\right)\right)^{2}},
$$

where

$$
L_{f}\left(x_{n}\right)=-\frac{1}{2} \frac{f^{\prime \prime}\left(x_{n}\right) f\left(x_{n}\right)}{f^{\prime}\left(x_{n}\right)+\lambda_{n} f\left(x_{n}\right)}
$$

and $\lambda_{n} \in \mathbb{R}, 0<\left|\lambda_{n}\right|<+\infty$ and $\mu_{n} \in \mathbb{R}, 0<\left|\mu_{n}\right|<+\infty$ with $n=0,1,2, \cdots$ are parameters. These parameters are chosen such that $\operatorname{sign}\left(\lambda_{n} f\left(x_{n}\right)\right)=$ $\operatorname{sign}\left(f^{\prime}\left(x_{n}\right)\right)$ and $\operatorname{sign}\left(\mu_{n} f\left(x_{n}\right)\right)=\operatorname{sign}\left(L_{f}\left(x_{n}\right)+f^{\prime}\left(x_{n}\right)\right)$, where

$$
\operatorname{sign}(x)= \begin{cases}1 & \text { if } x \geq 0 \\ -1 & \text { if } x<0\end{cases}
$$

is the sign function. The scheme (2) may be viewed as a new variant of well known Halley's method [11] with cubic convergence, but different from Halley's method, $f^{\prime}(x)=0$ in some points is permitted. For $(2)$, we have

Theorem 1 Assume that the function $f: D \subset \mathbb{R} \rightarrow \mathbb{R}$ for an open interval $D$ has a simple root $x^{*} \in D$. If $f(x)$ has first, second and third derivatives in the interval $D$, then the method defined by (2) converges cubically to $x^{*}$ in a neighborhood of $x^{*}$.

Proof. Let $e_{n}=x_{n}-x^{*}$. Using Taylor expansion, we have

$$
f\left(x^{*}\right)=f\left(x_{n}\right)-f^{\prime}\left(x_{n}\right) e_{n}+\frac{1}{2} f^{\prime \prime}\left(x_{n}\right) e_{n}^{2}-\frac{1}{6} f^{(3)}\left(x_{n}\right) e_{n}^{3}+O\left(e_{n}^{4}\right)
$$


Taking into account $f\left(x^{*}\right)=0$, we have

$$
f\left(x_{n}\right)=f^{\prime}\left(x_{n}\right) e_{n}-\frac{1}{2} f^{\prime \prime}\left(x_{n}\right) e_{n}^{2}+\frac{1}{6} f^{(3)}\left(x_{n}\right) e_{n}^{3}+O\left(e_{n}^{4}\right) .
$$

Furthermore, we have

$$
L_{f}\left(x_{n}\right)=-\frac{1}{2} \frac{f^{\prime \prime}\left(x_{n}\right)\left(f^{\prime}\left(x_{n}\right) e_{n}-\frac{1}{2} f^{\prime \prime}\left(x_{n}\right) e_{n}^{2}+O\left(e_{n}^{3}\right)\right)}{f^{\prime}\left(x_{n}\right)+\lambda_{n} f\left(x_{n}\right)} .
$$

From the relation

$$
\frac{f^{\prime}\left(x_{n}\right)}{f^{\prime}\left(x_{n}\right)+\lambda_{n} f\left(x_{n}\right)}=1-\frac{\lambda_{n} f\left(x_{n}\right)}{f^{\prime}\left(x_{n}\right)+\lambda_{n} f\left(x_{n}\right)}=1-\frac{\lambda_{n} f^{\prime}\left(x_{n}\right) e_{n}+O\left(e_{n}^{2}\right)}{f^{\prime}\left(x_{n}\right)+\lambda_{n} f\left(x_{n}\right)}
$$

the equation (4) becomes

$$
L_{f}\left(x_{n}\right)=-\frac{1}{2} f^{\prime \prime}\left(x_{n}\right) e_{n}+\frac{1}{2} \frac{f^{\prime \prime}\left(x_{n}\right)\left(\lambda_{n} f^{\prime}\left(x_{n}\right) e_{n}^{2}+\frac{1}{2} f^{\prime \prime}\left(x_{n}\right) e_{n}^{2}+O\left(e_{n}^{3}\right)\right)}{f^{\prime}\left(x_{n}\right)+\lambda_{n} f\left(x_{n}\right)} .
$$

Thus, we have

$$
\begin{aligned}
L_{f}\left(x_{n}\right) e_{n}+f^{\prime}\left(x_{n}\right) e_{n}-f\left(x_{n}\right)= & \frac{1}{2} \frac{f^{\prime \prime}\left(x_{n}\right)\left(\lambda_{n} f^{\prime}\left(x_{n}\right)+\frac{1}{2} f^{\prime \prime}\left(x_{n}\right)\right)}{f^{\prime}\left(x_{n}\right)+\lambda_{n} f\left(x_{n}\right)} e_{n}^{3} \\
& -\frac{1}{6} f^{(3)}\left(x_{n}\right) e_{n}^{3}+O\left(e_{n}^{4}\right)
\end{aligned}
$$

Since we have

$$
\begin{aligned}
& \frac{\left(L_{f}\left(x_{n}\right)+f^{\prime}\left(x_{n}\right)+\frac{1}{2} \mu_{n} f\left(x_{n}\right)\right)^{2}}{L_{f}\left(x_{n}\right)+f^{\prime}\left(x_{n}\right)+\mu_{n} f\left(x_{n}\right)}= \\
& L_{f}\left(x_{n}\right)+f^{\prime}\left(x_{n}\right)+\frac{1}{4} \frac{\mu_{n}^{2} f\left(x_{n}\right)^{2}}{L_{f}\left(x_{n}\right)+f^{\prime}\left(x_{n}\right)+\mu_{n} f\left(x_{n}\right)},
\end{aligned}
$$

the equation (2) can be expressed as

$$
\begin{aligned}
& x_{n+1}=x_{n}-\frac{f\left(x_{n}\right)}{L_{f}\left(x_{n}\right)+f^{\prime}\left(x_{n}\right)+\frac{1}{4} \frac{\mu_{n}^{2} f\left(x_{n}\right)^{2}}{L_{f}\left(x_{n}\right)+f^{\prime}\left(x_{n}\right)+\mu_{n} f\left(x_{n}\right)}}, \\
& e_{n+1}=e_{n}-\frac{f\left(x_{n}\right)}{L_{f}\left(x_{n}\right)+f^{\prime}\left(x_{n}\right)+\frac{1}{4} \frac{\mu_{n}^{2} f\left(x_{n}\right)^{2}}{L_{f}\left(x_{n}\right)+f^{\prime}\left(x_{n}\right)+\mu_{n} f\left(x_{n}\right)}}, \\
& e_{n+1}=\frac{L_{f}\left(x_{n}\right) e_{n}+f^{\prime}\left(x_{n}\right) e_{n}-f\left(x_{n}\right)+\frac{1}{4} \frac{\mu_{n}^{2} f\left(x_{n}\right)^{2} e_{n}}{L_{f}\left(x_{n}\right)+f^{\prime}\left(x_{n}\right)+\mu_{n} f\left(x_{n}\right)}}{L_{f}\left(x_{n}\right)+f^{\prime}\left(x_{n}\right)+\frac{1}{4} \frac{\mu_{n}^{2} f\left(x_{n}\right)^{2}}{L_{f}\left(x_{n}\right)+f^{\prime}\left(x_{n}\right)+\mu_{n} f\left(x_{n}\right)}} .
\end{aligned}
$$


Substituting (5) into (6) and using $f\left(x_{n}\right)^{2} e_{n}=f^{\prime}\left(x_{n}\right)^{2} e_{n}^{3}$, we have

$$
e_{n+1}=\frac{\left[\frac{f^{\prime \prime}\left(x_{n}\right)\left(\lambda_{n} f^{\prime}\left(x_{n}\right)+\frac{1}{2} f^{\prime \prime}\left(x_{n}\right)\right)}{2\left(f^{\prime}\left(x_{n}\right)+\lambda_{n} f\left(x_{n}\right)\right)}-\frac{1}{6} f^{(3)}\left(x_{n}\right)+\frac{1}{4} \frac{\mu_{n}^{2} f^{\prime}\left(x_{n}\right)^{2}}{L_{f}\left(x_{n}\right)+f^{\prime}\left(x_{n}\right)+\mu_{n} f\left(x_{n}\right)}\right] e_{n}^{3}+O\left(e_{n}^{4}\right)}{L_{f}\left(x_{n}\right)+f^{\prime}\left(x_{n}\right)+\frac{1}{4} \frac{\mu_{n}^{2} f\left(x_{n}\right)^{2}}{L_{f}\left(x_{n}\right)+f^{\prime}\left(x_{n}\right)+\mu_{n} f\left(x_{n}\right)}} .
$$

Therefore, we have

$$
\lim _{n \rightarrow+\infty} \frac{e_{n+1}}{e_{n}^{3}}=\frac{1}{4} \mu^{2}+\frac{1}{2} \lambda \frac{f^{\prime \prime}\left(x^{*}\right)}{f^{\prime}\left(x^{*}\right)}+\frac{1}{4} \frac{f^{\prime \prime}\left(x^{*}\right)^{2}}{f^{\prime}\left(x^{*}\right)^{2}}-\frac{1}{6} \frac{f^{(3)}\left(x^{*}\right)}{f^{\prime}\left(x^{*}\right)} .
$$

This means that the method defined by (2) is cubically convergent.

\section{Second-derivative-free methods}

In $(2)$, the severe condition $f^{\prime}(x) \neq 0$ in some neighborhood of the root $x^{*}$ is removed, but the second derivative $f^{\prime \prime}(x)$ is yet required and the required methods are not derived. So we will present a class of new second-derivative-free iterative methods, based on iterative formula (2) in which the second derivative is replaced with a finite difference quotient between the first derivatives.

Now, we consider a finite difference quotient

$$
f^{\prime \prime}\left(x_{n}\right) \simeq \frac{f^{\prime}\left(y_{n}\right)-f^{\prime}\left(x_{n}\right)}{y_{n}-x_{n}},
$$

where

$$
y_{n}=x_{n}-\alpha \frac{f\left(x_{n}\right)}{f^{\prime}\left(x_{n}\right)+\lambda_{n} f\left(x_{n}\right)}, \alpha \in \mathbb{R}, \alpha \neq 0 .
$$

Then, from (2) we obtain a class of new iterative methods free from second derivatives

$$
x_{n+1}=x_{n}-\frac{\left(H\left(\alpha, x_{n}\right)+\mu_{n} f\left(x_{n}\right)\right) f\left(x_{n}\right)}{\left(H\left(\alpha, x_{n}\right)+\frac{1}{2} \mu_{n} f\left(x_{n}\right)\right)^{2}},
$$

where $H\left(\alpha, x_{n}\right)=\frac{1}{2 \alpha} f^{\prime}\left(y_{n}\right)+\left(1-\frac{1}{2 \alpha}\right) f^{\prime}\left(x_{n}\right)$ and $y_{n}$ is defined by (7). The parameters $\lambda_{n}$ and $\mu_{n}$ are chosen such that $\operatorname{sign}\left(\lambda_{n} f\left(x_{n}\right)\right)=\operatorname{sign}\left(f^{\prime}\left(x_{n}\right)\right)$ and $\operatorname{sign}\left(\mu_{n} f\left(x_{n}\right)\right)=\operatorname{sign}\left(H\left(\alpha, x_{n}\right)\right)$ respectively.

If considering the different values of the parameter $\alpha$ in (8), we can obtain a class of new iterative methods that includes, as particular cases, the following 
ones:

1. As a limit case, when $\alpha \rightarrow 0$, the method defined by (2) is obtained.

2. For $\alpha=1$, we obtain a new third-order method

$$
x_{n+1}=x_{n}-\frac{\left(H\left(1, x_{n}\right)+\mu_{n} f\left(x_{n}\right)\right) f\left(x_{n}\right)}{\left(H\left(1, x_{n}\right)+\frac{1}{2} \mu_{n} f\left(x_{n}\right)\right)^{2}},
$$

where $H\left(1, x_{n}\right)=\left(f^{\prime}\left(y_{n}\right)+f^{\prime}\left(x_{n}\right)\right) / 2$ and $y_{n}=x_{n}-f\left(x_{n}\right) /\left(f^{\prime}\left(x_{n}\right)+\lambda_{n} f\left(x_{n}\right)\right)$. This method is the required variant of the method presented in [2]

$$
x_{n+1}=x_{n}-\frac{2 f\left(x_{n}\right)}{f^{\prime}\left(x_{n}-\frac{f\left(x_{n}\right)}{f^{\prime}\left(x_{n}\right)}\right)+f^{\prime}\left(x_{n}\right)} .
$$

3. For $\alpha=\frac{1}{2}$, we obtain another new third-order method

$$
x_{n+1}=x_{n}-\frac{\left(f^{\prime}\left(y_{n}\right)+\mu_{n} f\left(x_{n}\right)\right) f\left(x_{n}\right)}{\left(f^{\prime}\left(y_{n}\right)+\frac{1}{2} \mu_{n} f\left(x_{n}\right)\right)^{2}},
$$

where $y_{n}=x_{n}-\frac{1}{2} f\left(x_{n}\right) /\left(f^{\prime}\left(x_{n}\right)+\lambda_{n} f\left(x_{n}\right)\right)$. This method is the required variant of the method presented in [3-5]

$$
x_{n+1}=x_{n}-\frac{f\left(x_{n}\right)}{f^{\prime}\left(x_{n}-\frac{1}{2} \frac{f\left(x_{n}\right)}{f^{\prime}\left(x_{n}\right)}\right)} .
$$

We can see that iterative formulae (9) and (11) can remove the severe condition $f^{\prime}(x) \neq 0$ which is necessary to iterative formulae (10) and (12). Per iteration the new methods defined by (8), including iterative formulae (9) and (11) , also require one evaluation of the function and two of its first derivative, but no evaluations of its second derivative. Thus, the new methods may be preferable in more cases, especially where sufficiently good initial approximations to the required root is not provided or the first derivative $f^{\prime}(x)$ is small or even equal to zero in a neighborhood of the required root. For (8), we have

Theorem 2 Under the conditions of Theorem 1, the methods defined by (8) converge cubically to $x^{*}$ in a neighborhood of $x^{*}$.

Proof. Let $e_{n}=x_{n}-x^{*}$ and $d_{n}=y_{n}-x_{n}$, where $y_{n}$ is defined by (7). Similar to the proof of Theorem 1 , we have the equation (3) and then we have

$$
d_{n}=-\alpha \frac{f\left(x_{n}\right)}{f^{\prime}\left(x_{n}\right)+\lambda_{n} f\left(x_{n}\right)}
$$




$$
\begin{aligned}
& =-\alpha \frac{f^{\prime}\left(x_{n}\right) e_{n}-\frac{1}{2} f^{\prime \prime}\left(x_{n}\right) e_{n}^{2}}{f^{\prime}\left(x_{n}\right)+\lambda_{n} f\left(x_{n}\right)}+O\left(e_{n}^{3}\right) \\
& =-\alpha e_{n}+\alpha \frac{\lambda_{n} f^{\prime}\left(x_{n}\right)+\frac{1}{2} f^{\prime \prime}\left(x_{n}\right)}{f^{\prime}\left(x_{n}\right)+\lambda_{n} f\left(x_{n}\right)} e_{n}^{2}+O\left(e_{n}^{3}\right) .
\end{aligned}
$$

Expanding $f^{\prime}\left(y_{n}\right)$ about $x_{n}$, we have

$$
f^{\prime}\left(y_{n}\right)=f^{\prime}\left(x_{n}\right)+f^{\prime \prime}\left(x_{n}\right) d_{n}+\frac{1}{2} f^{(3)}\left(x_{n}\right) d_{n}^{2}+O\left(d_{n}^{3}\right)
$$

and then from (13), we have

$$
\begin{aligned}
f^{\prime}\left(y_{n}\right)= & f^{\prime}\left(x_{n}\right)-\alpha f^{\prime \prime}\left(x_{n}\right) e_{n}+\alpha \frac{\lambda_{n} f^{\prime}\left(x_{n}\right) f^{\prime \prime}\left(x_{n}\right)+\frac{1}{2} f^{\prime \prime}\left(x_{n}\right)^{2}}{f^{\prime}\left(x_{n}\right)+\lambda_{n} f\left(x_{n}\right)} e_{n}^{2} \\
& +\frac{1}{2} \alpha^{2} f^{(3)}\left(x_{n}\right) e_{n}^{2}+O\left(e_{n}^{3}\right) .
\end{aligned}
$$

Thus, we have

$$
\begin{aligned}
H\left(\alpha, x_{n}\right)= & f^{\prime}\left(x_{n}\right)-\frac{1}{2} f^{\prime \prime}\left(x_{n}\right) e_{n}+\frac{1}{2} \frac{\lambda_{n} f^{\prime}\left(x_{n}\right) f^{\prime \prime}\left(x_{n}\right)+\frac{1}{2} f^{\prime \prime}\left(x_{n}\right)^{2}}{f^{\prime}\left(x_{n}\right)+\lambda_{n} f\left(x_{n}\right)} e_{n}^{2} \\
& +\frac{1}{4} \alpha f^{(3)}\left(x_{n}\right) e_{n}^{2}+O\left(e_{n}^{3}\right) .
\end{aligned}
$$

Since from (8) we have

$$
\begin{aligned}
e_{n+1} & =e_{n}-\frac{f\left(x_{n}\right)}{H\left(\alpha, x_{n}\right)+\frac{1}{4} \frac{\mu_{n}^{2} f\left(x_{n}\right)^{2}}{H\left(\alpha, x_{n}\right)+\mu_{n} f\left(x_{n}\right)}} \\
& =\frac{H\left(\alpha, x_{n}\right) e_{n}+\frac{1}{4} \frac{\mu_{n}^{2} f\left(x_{n}\right)^{2}}{H\left(\alpha, x_{n}\right)+\mu_{n} f\left(x_{n}\right)} e_{n}-f\left(x_{n}\right)}{H\left(\alpha, x_{n}\right)+\frac{1}{4} \frac{\mu_{n}^{2} f\left(x_{n}\right)^{2}}{H\left(\alpha, x_{n}\right)+\mu_{n} f\left(x_{n}\right)}}
\end{aligned}
$$

using $f\left(x_{n}\right)^{2} e_{n}=f^{\prime}\left(x_{n}\right)^{2} e_{n}^{3}$ and from (3) and (15), we have

$$
e_{n+1}=\frac{\left[\frac{f^{\prime \prime}\left(x_{n}\right)\left(\lambda_{n} f^{\prime}\left(x_{n}\right)+\frac{1}{2} f^{\prime \prime}\left(x_{n}\right)\right)}{2\left(f^{\prime}\left(x_{n}\right)+\lambda_{n} f\left(x_{n}\right)\right)}+\left(\frac{\alpha}{4}-\frac{1}{6}\right) f^{(3)}\left(x_{n}\right)+\frac{1}{4} \frac{\mu_{n}^{2} f^{\prime}\left(x_{n}\right)^{2}}{H\left(\alpha, x_{n}\right)+\mu_{n} f\left(x_{n}\right)}\right] e_{n}^{3}+O\left(e_{n}^{4}\right)}{H\left(\alpha, x_{n}\right)+\frac{1}{4} \frac{\mu_{n}^{2} f\left(x_{n}\right)^{2}}{H\left(\alpha, x_{n}\right)+\mu_{n} f\left(x_{n}\right)}} .
$$

Therefore, we have

$$
\lim _{n \rightarrow+\infty} \frac{e_{n+1}}{e_{n}^{3}}=\frac{1}{4} \mu^{2}+\frac{1}{2} \lambda \frac{f^{\prime \prime}\left(x^{*}\right)}{f^{\prime}\left(x^{*}\right)}+\frac{1}{4} \frac{f^{\prime \prime}\left(x^{*}\right)^{2}}{f^{\prime}\left(x^{*}\right)^{2}}+\left(\frac{\alpha}{4}-\frac{1}{6}\right) \frac{f^{(3)}\left(x^{*}\right)}{f^{\prime}\left(x^{*}\right)} .
$$

This means that the methods defined by (8) are cubically convergent. 


\section{Numerical examples}

Now we employ new iterative methods (9) and (11) with $\left|\lambda_{n}\right|=\left|\mu_{n}\right|=1$ to solve some non-linear equations and compare these methods with iterative formulae (10) and (12). The computational results are displayed in Tables 1 and 2 respectively. From examples 1-3, we can see that iterative formulae (9) and (11) can remove the severe condition $f^{\prime}(x) \neq 0$. From examples 4-5, we can see that iterative formulae (9) and (11) are possible in global convergence. Therefore, the new methods may succeed in convergence in case the initial approximation is far from the required root or the derivative is small or even equal to zero in a neighborhood of the required root.

Example 1. $f(x)=x^{3}-3 x+1, \quad x_{0}=1$.

Example 2. $f(x)=x \sin (x)+\cos (x)-1.5, \quad x_{0}=0$.

Example 3. $f(x)=e^{-x}+2 \sin (x)-x+3, \quad x_{0}=0$.

Example 4. $f(x)=\ln (x-1), \quad x_{0}=16$.

Example 5. $f(x)=\arctan (x), \quad x_{0}=8$.

Table 1. Comparison of iterative formulae (9) and (10)

\begin{tabular}{lllll}
\hline Example & \multicolumn{3}{c}{ Iterative method $(9)$} & \multirow{2}{*}{$\begin{array}{l}\text { Iterative } \\
\text { (10) }\end{array}$} \\
\cline { 2 - 4 } & $n$ & $x_{n}$ & $\left|f\left(x_{n}\right)\right|$ & \\
\hline 1 & 3 & 0.3472963553338606 & $2.22 \mathrm{e}-16$ & divergence \\
2 & 4 & -1.2460765069513031 & $2.22 \mathrm{e}-16$ & failure \\
3 & 4 & 3.1092692039941778 & $8.88 \mathrm{e}-15$ & failure \\
4 & 8 & 1.9999999999999645 & $3.55 \mathrm{e}-14$ & divergence \\
5 & 5 & $-8.27181 \mathrm{e}-25$ & $8.27 \mathrm{e}-25$ & divergence \\
\hline
\end{tabular}

Table 2. Comparison of iterative formulae (11) and (12)

\begin{tabular}{lllll}
\hline Example & \multicolumn{3}{c}{ Iterative method (11) } & \multirow{2}{*}{$\begin{array}{l}\text { Iterative } \\
\text { (12) }\end{array}$} \\
\cline { 2 - 4 } & $n$ & $x_{n}$ & $\left|f\left(x_{n}\right)\right|$ & \\
\hline 1 & 4 & 0.3472963553338607 & $2.22 \mathrm{e}-16$ & divergence \\
2 & 4 & -1.2460765069513000 & $1.11 \mathrm{e}-15$ & failure \\
3 & 4 & 3.1092692039941796 & $3.55 \mathrm{e}-15$ & failure \\
4 & 7 & 1.9999999999999998 & $2.22 \mathrm{e}-16$ & divergence \\
5 & 5 & $1.48866 \mathrm{e}-19$ & $1.49 \mathrm{e}-19$ & divergence \\
\hline
\end{tabular}




\section{Conclusions}

We have obtained a class of new third-order methods free from second derivative for solving the problem mentioned in Section 1. This class of new methods removes the severe condition $f^{\prime}(x) \neq 0$ in a neighborhood of the root. Thus, these new methods may succeed in convergence in case sufficiently good initial approximations to the required root is not provided or the derivative is small or even equal to zero in a neighborhood of the required root. From a practical point of view, the new methods have the definite practical utility.

\section{Acknowledgement}

Work supported by National Natural Science Foundation of China (50379038; 40474003).

\section{References}

[1] A.M. Ostrowski, Solution of Equations in Eucilidean and Banach Space, 3rd ed., Academic Press, New York (1973).

[2] S. Weerakoon and T.G.I. Fernando, A variant of Newton's method with accelerated third-order convergence, Appl. Math. Lett., 13 (2000), 87-93.

[3] M. Frontini and E. Sormani, Some variants of Newton's method with third-order convergence, Appl. Math. Comput., 140 (2003), 419-426.

[4] A.Y. Özban, Some new variants of Newton's method, Appl. Math. Lett., 17 (2004), 677-682.

[5] H.H.H. Homeier, A modified Newton method for rootfinding with cubic convergence, J. Comput. Appl. Math., 157 (2003), 227-230.

[6] M. Frontini and E. Sormani, Modified Newton's method with third-order convergence and multiple roots, J. Comput. Appl. Math., 156 (2003), 345-354. 
[7] H.H.H. Homeier, A modified Newton method with cubic convergence: the multivariate case, J. Comput. Appl. Math., 169 (2004), 161-169.

[8] M. Frontini and E. Sormani, Third-order methods from quadrature formulae for solving systems of nonlinear equations, Appl. Math. Comput., 149 (2004), 771-782.

[9] S. Amat, S. Busquier and J.M. Gutiérrez, Geometric constructions of iterative functions to solve nonlinear equations, J. Comput. Appl. Math., 157 (2003), 197-205.

[10] X.Y. Wu, A new continuation Newton-like method and its deformation, Appl. Math. Comput., 112 (2000), 75-78.

[11] I.K. Argyros, A note on the Halley method in Banach spaces, Appl. Math. Comput., 58 (1993), 215-224.

Received: March 28, 2006 